\title{
A protein crystallization strategy for structure-based drug design
}

\section{Terese Bergfors*}

*Dept. of Cell and Molecular Biology, Uppsala University, Sweden; terese.bergfors@icm.uu.se

STOP (Same-Target-Other-Pathogens) is our structure-based drug-discovery effort that targets proteins in the non-mevalonate pathway of multiple pathogens (1). These include Mycobacterium tuberculosis (tuberculosis); Plasmodium falciparum and P. vivax (malaria); and the ESKAPE bacteria (a group implicated in hospital-acquired infections).

At STOP we use a minimalist approach to screen for crystallization conditions. How well does this approach work? I will present our experiences with it and also discuss choosing a screen, protein formulation, and seeding with homologues (2), Fig. 1.

Our experiences at STOP may be relevant to other small academic and medium-throughput laboratories. The lecture will also be of interest to researchers interested in crystallization strategy in general.
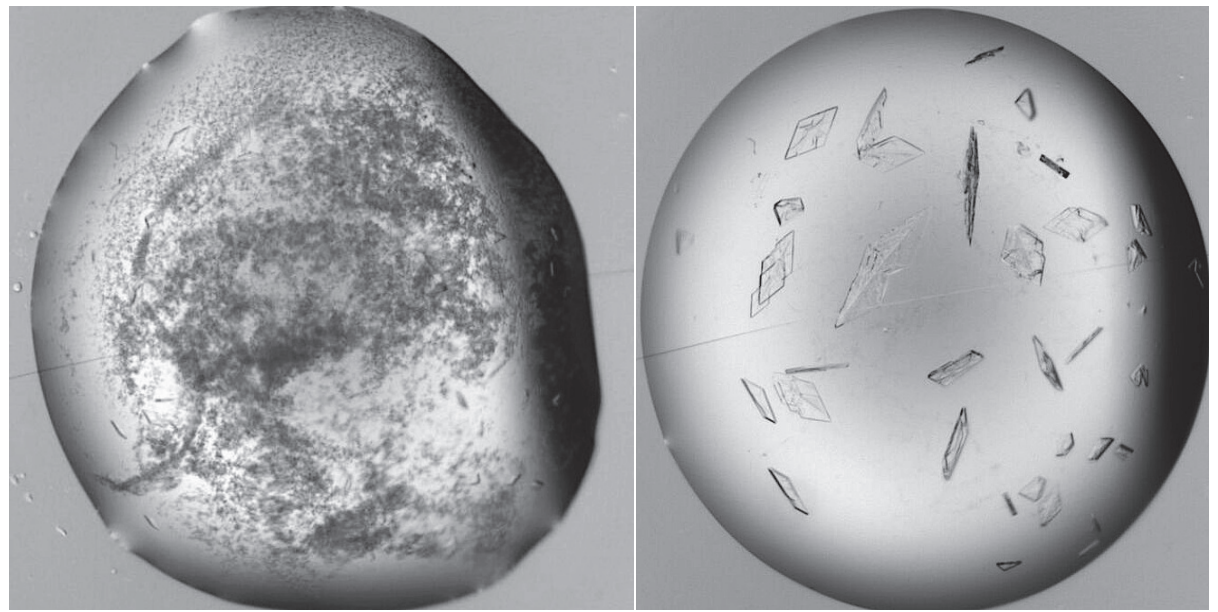

Fig. 1. Cross-seeding of Plasmodium falciparum IspC. Crystals were difficult to obtain until the drops were cross-seeded with crystals from a homologue, the $P$. vivax IspC. The homologues have $70 \%$ sequence identity. The micrographs show the droplets with $P$. falciparum IspC protein at 1 hour (left) and 12 hours (right) after seeding with $P$. vivax IspC crystals. The crystals on the right are $0.15 \mathrm{~mm}$ in their longest direction and diffract to $1.6 \AA$.

\section{References}

1. Sooriyaarachchi, S., et al. (2016) ChemMedChem 11, 1-14.

2. D'Arcy, A., Bergfors, T., Cowan-Jacob, S.W. and Marsh, M. (2014) Acta Cryst F70, 1117-1126. 